\title{
Searching iron sensors in plants by exploring the link among 2'-OG-dependent dioxygenases, the iron deficiency response and metabolic adjustments occurring under iron deficiency
}

\author{
Gianpiero Vigani ${ }^{1}$, Piero Morandini ${ }^{2}$ and Irene Murgia ${ }^{2}$ * \\ ' Dipartimento di Scienze Agrarie ed Ambientali-Produzioni, Territorio, Agroenergia, Università degli Studi di Milano, Milano, Italy \\ 2 Dipartimento di Bioscienze, Università degli Studi di Milano, Milano, Italy
}

\author{
Edited by: \\ Jean-Francois Briat, Centre National \\ de la Recherche Scientifique, France \\ Reviewed by: \\ Wolfgang Schmidt, Academia Sinica, \\ Taiwan \\ Sabine Lüthje, University of Hamburg, \\ Germany

\section{${ }^{*}$ Correspondence:} \\ Irene Murgia, Dipartimento di \\ Bioscienze, Università degli Studi di \\ Milano, Via Celoria 26, 20133 Milano, \\ Italy \\ e-mail: irene.murgia@unimi.it
}

\begin{abstract}
Knowledge accumulated on the regulation of iron $(\mathrm{Fe})$ homeostasis, its intracellular trafficking and transport across various cellular compartments and organs in plants; storage proteins, transporters and transcription factors involved in Fe metabolism have been analyzed in detail in recent years. However, the key sensor(s) of cellular plant "Fe status" triggering the long-distance shoot-root signaling and leading to the root Fe deficiency responses is (are) still unknown. Local Fe sensing is also a major task for roots, for adjusting the internal Fe requirements to external Fe availability: how such sensing is achieved and how it leads to metabolic adjustments in case of nutrient shortage, is mostly unknown. Two proteins belonging to the 2'-OG-dependent dioxygenases family accumulate several folds in Fe-deficient Arabidopsis roots. Such proteins require Fe(II) as enzymatic cofactor; one of their subgroups, the HIF-P4H (hypoxia-inducible factor-prolyl 4-hydroxylase), is an effective oxygen sensor in animal cells. We envisage here the possibility that some members of the 2'-OG dioxygenase family may be involved in the Fe deficiency response and in the metabolic adjustments to Fe deficiency or even in sensing Fe, in plant cells.
\end{abstract}

Keywords: Arabidopsis thaliana, iron sensor, HIF (hypoxia-inducible factor), 2'-OG-dependent dioxygenase, prolyl 4-hydroxylase

\section{INTRODUCTION}

Iron is an essential micronutrient for plants although it is potentially toxic, when present in a free, non-complexed form. A recent review on that subject (Kobayashi and Nishizawa, 2012) details the knowledge accumulated on the regulation of plant Fe homeostasis, its intracellular trafficking and transport across cellular compartments and organs under various conditions of Fe supply, unveiling a complex net of molecular interactions. Beside the intensification of Fe-uptake strategies activated by plants under Fe-limiting conditions, root cells reprogram their metabolism to better cope with shortage of Fe (Vigani et al., 2012). Low Fe content triggers a high energy request to sustain the increased rate of Fe uptake from the soil, and at the same time it impairs the function of mitochondria and chloroplasts which provide energy to the cells. Thus, cells must increase the rate of alternative energy-providing pathways, such as glycolysis, Krebs cycle, or pentose phosphate pathway (LópezMillán et al., 2000, 2012; Li et al., 2008; Vigani and Zocchi, 2009; Donnini et al., 2010; Rellán-Álvarez et al., 2010; Vigani, 2012a). To date, however, the sensors of plant "Fe status" triggering the signal transduction pathways, which eventually induce transcription factors such as the Arabidopsis FIT1, are still unknown and represent a challenging issue in plant science (Vigani et al., 2013). Efforts to fill up such gap of knowledge have been made by different research groups since years (Schmidt and Steinbach, 2000); recently, it has been demonstrated that localized Fe supply stimulates lateral root formation through the AUX1 auxin importer, which is proposed as a candidate for integrating the local Fe status in auxin signaling (Giehl et al., 2012).

\section{2'-OG Fe(II)-DEPENDENT DIOXYGENASES AND PROLYL 4-HYDROXYLASES}

It has been recently observed that some similarities might exist between the metabolic reprogramming occurring in Fe-deficient roots and that one occurring in tumor cells (Vigani, 2012b). In tumor cells, such reprogramming is known as "Warburg-effect" in which glucose is preferentially converted to lactate by enhancing glycolysis and fermentative reactions rather than completely oxidized by oxidative phosphorylation (OXOPHOS; Brahimi-Horn et al., 2007). Also in root cells a low Fe availability causes a decrease of OXOPHOS activity and induction of glycolysis and anaerobic reactions (Vigani, 2012b). The Warburg-effect in animal cells is mediated by hypoxia-inducible factor (HIF1), a heterodimeric complex whose $\alpha$ subunit is inducible by hypoxia. Under normoxic conditions, HIF $\alpha$ is post-translationally modified via the hydroxylation of proline residues by prolyl 4-hydroxylases $(\mathrm{P} 4 \mathrm{H})$; such modification leads to the proteasome-mediated degradation of HIF $\alpha$. Under hypoxic conditions, however, such hydroxylation cannot occur because $\mathrm{P} 4 \mathrm{H}$ enzymes belong to the 2-oxoglutarate $\mathrm{Fe}(\mathrm{II})$-dependent dioxygenase family which have molecular oxygen and oxoglutarate as co-substrates; in other words, the lack of oxygen inhibits the $\mathrm{P} 4 \mathrm{H}$ enzymatic activity, $\mathrm{HIF} \alpha$ escapes degradation, it translocates to the nucleus where it can therefore form a 
dimer with HIF $\beta$ subunit; the complex then activates the cascade of hypoxia-responsive gene expression pathways (Myllyharju, 2003; Ken and Costa, 2006; Semenza, 2007).

Prolyl 4-hydroxylases are present in animal as well as in plant cells. In animal cells, $\mathrm{P} 4 \mathrm{H}$ are classified into two categories: the collagen-type-P4H and the above cited HIF-P4H. The first class is localized within the lumen of the endoplasmic reticulum and it catalyzes the hydroxylation of proline residues within -X-Pro-Glysequences in collagen and in collagen-type proteins (Myllyharju, 2003), thus stabilizing their triple helical structure at body temperature (Myllyharju, 2003). These $\mathrm{P} 4 \mathrm{Hs}$ are $\alpha_{2} \beta_{2}$ tetramers and their catalytic site is located in the $\alpha$ subunit (Myllyharju, 2003; Tiainen et al., 2005). Three aa residues, His-412, Asp-414, and His-483, are the binding sites for $\mathrm{Fe}(\mathrm{II})$ in the human $\alpha(\mathrm{I})$ subunit (Myllyharju, 2003). The second class of $\mathrm{P} 4 \mathrm{H}$ is localized in cytoplasm and it is responsible for hydroxylation of a proline residue in the HIFa subunit, under normoxic conditions, as described above. The $K_{\mathrm{m}}$ values of HIF-P4Hs for $\mathrm{O}_{2}$ are slightly above atmospheric concentration, making such proteins effective $\mathrm{O}_{2}$ sensors (Hirsilä et al., 2003). A novel role has also been uncovered for a human collagen-type- $\mathrm{P} 4 \mathrm{H}$, as regulator of Argonaute2 stability with consequent influence on RNA interference mechanisms (Qi et al., 2008).

Several genes similar to $\mathrm{P} 4 \mathrm{H}$ are present in plants; for instance, $13 \mathrm{P} 4 \mathrm{H}$ have been identified in Arabidopsis and named AtP4H1-AtP4H13 (Vlad et al., 2007a,b); with the exception of AtP4H11 and AtP4H12, the three binding residues for Fe(II) (two His and one Asp) as well as the Lys residues binding the 2-oxoglutarate, are all conserved in such $\mathrm{P} 4 \mathrm{Hs}$ (Vlad et al., 2007a). The different isoforms are more expressed in roots than in shoots and they show different pattern of expression in response to various stresses (hypoxia, anoxia, and mechanical wounding; Vlad et al., 2007a,b).

Cloning and biochemical characterization of two of them, i.e., AtP4H1, encoded by At2g43080 gene (Hieta and Myllyharju, 2002) and At4PH2, encoded by At3g06300 gene (Tiainen et al., 2005) show that substrate specificity varies: recombinant AtP4H1 effectively hydroxylates poly(L-proline) and other synthetic peptides with $K_{\mathrm{m}}$ values lower than those for AtP4H2, thus suggesting different physiological roles between the two. Recombinant AtP4H1 can also effectively hydroxylate human HIF $\alpha$-like peptides and collagen-like peptides, whereas recombinant AtP4H2 cannot (Hieta and Myllyharju, 2002; Tiainen et al., 2005). Their $K_{\mathrm{m}}$ for $\mathrm{Fe}(\mathrm{II})$ are 16 and $5 \mu \mathrm{M}$, respectively (Hieta and Myllyharju, 2002; Tiainen et al., 2005).

Two proteins belonging to the $2^{\prime}$-OG dioxygenase family, encoded by At3g12900 and At3g13610 genes, accumulate several folds in $\mathrm{Fe}$-deficient roots, when compared to $\mathrm{Fe}$-sufficient ones (Lan et al., 2011). The protein encoded by At3g13610 gene, named $\mathrm{F}^{\prime} \mathrm{H} 1$, is involved in the synthesis of coumarins via the phenylpropanoid pathway, as it catalyzes the ortho-hydroxylation of feruloyl CoA, which is the precursor of scopoletin (Kai et al., 2008). Scopoletin and its $\beta$-glucoside scopolin accumulate in Arabidopsis roots and, at lower levels, also in shoots (Kai et al., 2006).

A severe reduction of scopoletin levels can be observed in the KO mutants for the At3g13610 gene (Kai et al., 2008). One of the responses to Fe deficiency, is the induction of the phenylpropanoid pathway (Lan et al., 2011). Phenolics can facilitate the reutilization of root apoplastic Fe (Jin et al., 2007a,b) and a phenolic efflux transporter PEZ1 located in the stele has been identified in rice (Ishimaru et al., 2011). The secretion of phenolic compounds can, moreover, selectively modify the soil microbial population in the surroundings of the roots, which in turn can favor acquisition of Fe by production of siderophores as well as auxin-like compounds (Jin et al., 2006, 2008, 2010).

Plant 2'-OG dioxygenases are also involved in synthesis of phytosiderophores such as Ids3 from barley, which is induced by Fe deficiency and it catalyzes the hydroxylation step from $2^{\prime}$ deoxymugeinic acid (DMA) to mugeinic acid (MA; Kobayashi et al., 2001).

\section{2'-OG Fe(II)-DEPENDENT DIOXYGENASES, Fe DEFICIENCY RESPONSE AND METABOLIC REPROGRAMMING: IS THERE A COMMON LINK?}

Given the above premises, it is possible that a link among $\mathrm{P} 4 \mathrm{H}$ activity, and more generally among $2^{\prime}-\mathrm{OG} \mathrm{Fe}(\mathrm{II})$-dependent dioxygenase activities, the $\mathrm{Fe}$ deficiency responses and the metabolic reprogramming occurring during Fe deficiency exists in higher plants. If such a link exists for a given 2'-OG Fe(II)dependent dioxygenase, at least two possible scenarios could be predicted for such enzyme (Figure 1):

(a) If, for a given sub-cellular localization, the $K_{\mathrm{m}}$ of such enzyme for $\mathrm{Fe}$ is close to the physiological concentration of the LIP (labile iron pool, consisting of free redox-active Fe ions), then the enzyme activity is strongly affected by Fe fluctuations, similarly to the above described HIF-P4H, which is an effective sensor for $\mathrm{O}_{2}$ (Hirsilä et al., 2003). Upon reduction of Fe availability below the physiological LIP, its enzymatic activity should be indeed drastically reduced or fully inhibited; reduction or complete lack of enzymatic product might, in turn, triggers the "Fe deficiency" signaling. The enzyme might therefore act as true Fe sensor (Figure 1, upper panel, right).

Although the Fe-dependent transcriptional regulation of such an Fe sensor enzyme might be not expected, it cannot be excluded a priori: for example, chitin recognition is dependent not only on the presence of specific receptors, but also on the expression of extracellular chitinases, which are essential for the production of smaller chito-oligosaccharides from chitin hydrolysis, in animal (Gorzelanny et al., 2010; Vega and Kalkum, 2012) as well as in plant systems (Shibuya and Minami, 2001; Wan etal., 2008). These smaller, diffusible molecules induce, in turn, the expression of several defense protein, among which also chitinase activities. Chitinase is thus both an example of a crucial enzyme for the signal production but also an integral part of the response.

(b) If, for a given sub-cellular localization, the $K_{\mathrm{m}}$ for Fe of such an enzyme is instead far below the physiological LIP, the enzyme might still be active under Fe deficiency. Additionally, if transcriptional/translational up-regulation occurs under Fe deficiency, accumulation of protein and increased total enzymatic activity might be observed. The enzyme might be involved in the Fe response/metabolic adjustment occurring under Fe deficiency, without being itself a Fe sensor (Figure 1, lower panel, right). 


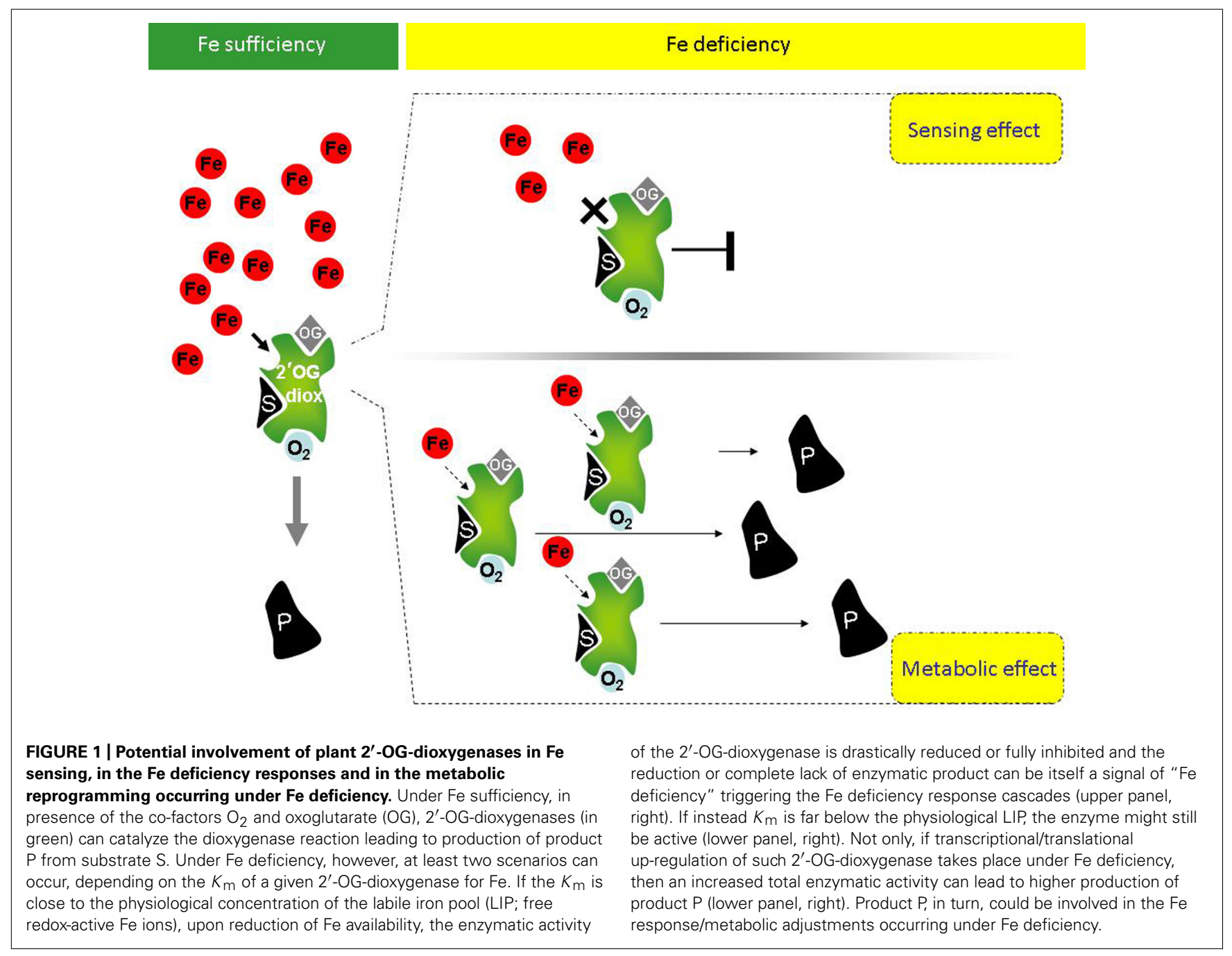

This second scenario is supported by the evidence that the Arabidopsis 2'-OG-dioxygenase $\mathrm{F}^{\prime} \mathrm{H} 1$ (described in previous paragraph) which accumulates in Fe-deficient roots (Lan et al., 2011) is indeed possibly involved in the Fe response/metabolic adjustment occurring under Fe deficiency: Arabidopsis mutants $\mathrm{KO}$ for the At3g13610 gene (coding for F6 ${ }^{\prime} \mathrm{H} 1$ ) have indeed altered root phenotype under Fe deficiency (I. Murgia, unpublished observations).

Such a link among $2^{\prime}$-OG Fe(II)-dependent dioxygenase activity, the Fe deficiency responses and the metabolic reprogramming occurring during Fe deficiency can be explored first by analyzing the transcriptional co-regulation of $2^{\prime}$-OG-dependent dioxygenase genes with genes involved in the Fe deficiency response or in the metabolic reprogramming. The bioinformatic approach of our choice was already described (Beekwilder et al., 2008; Menges et al., 2008; Berri et al., 2009; Murgia et al., 2011) and successfully applied in Arabidopsis and rice. Such analysis identifies genes which are co-regulated in large microarray datasets; in this case, it provides candidate genes potentially involved in Fe metabolism, among the 2 -OG-dioxygenase family members. Although transcript levels do not equal protein levels (or activities), there is

nevertheless evidence for correlation between the two in many organisms (Vogel and Marcotte, 2012). This approach is not only simple on a genomic scale, but it has proved useful to identify candidate genes in the past, which were then validated by experimental approaches (e.g., Beekwilder et al., 2008; Murgia et al., 2011; Møldrup et al., 2012).

Arabidopsis possesses almost one hundred annotated $2^{\prime}-\mathrm{OG}$ dioxygenase genes which make such analysis not immediate; we therefore restricted the analysis to the AtP4H subclass (with the exclusion of AtP4H8, AtP4H12, and AtP4H13 because the corresponding genes were not available in the Affymetrix microarray data set most commonly used). As pivot bioinformatic analysis, we analyzed the correlation of such AtP4H subclass with two gene groups. The first group consisted of a list of 25 Fe-homeostasis/trafficking/transport related genes, described in recent reviews on this subject (Conte and Walker, 2011; Kobayashi and Nishizawa, 2012). The second group consisted of an equal number of genes coding for enzymes possibly involved in the metabolic adjustments under Fe deficiency, such as those catalyzing the synthesis of pyruvate (Pyr). It is indeed known that several glycolitic genes are overexpressed in roots of Fe-deficient 
plants (Thimm etal., 2001): different isoforms of hexokinase (HXK), phosphoglyceratekinase, enolase (ENO), phosphoglycerate mutase (iPGAM), were therefore considered. Also, genes coding for enzymes involved in the consumption of Pyr by non-OXOPHOS reactions and whose expression is affected by Fe deficiency, such as alcohol dehydrogenase $(\mathrm{ADH})$, lactate dehydrogenase (LDH), and malate dehydrogenase, were also considered (Thimm et al., 2001). Last, the genes coding for the four isoforms of Arabidopsis phosphoenolpyruvate carboxylase (PEPC; PPC1, 2, 3, 4; Sanchez et al., 2006) were also included in the second group, since PEPC is strongly induced in several dicotyledonous plants under Fe deficiency (Vigani, 2012a) and PEPC is supposed to play a central role in the metabolic reprogramming occurring in Fe-deficient root cells (Zocchi, 2006).

As positive controls, the two 2'-OG-dioxygenases encoded by At3g12900 and At3g13610 and accumulating in Fe-deficient roots (Lan et al., 2011) whereas, as negative control, the ferritin gene whose expression is known to be repressed under Fe deficiency (Murgia et al., 2002), were included. The full list of genes for which the correlation analysis has been performed, is reported in Table 1.

The resulting Pearson's correlation coefficients, calculated by using either linear or logarithmic expression values (Menges et al., 2008; Murgia etal., 2011) are reported in Table 2, if above a defined threshold $(\geq 0.60$ or $\leq-0.60)$; genes for which none of the Pearson's coefficient fulfilled this condition, were not included in Table 2 (AtP4H3, AtP4H9, AtP4H10 and AtP4H11).

In accordance with results obtained by iTRAQ (isobaric peptide tags for relative and absolute quantitation) analysis of Fe-deficient roots (Lan etal., 2011), both At3g12900 and At3g136100 show positive correlation with genes actively involved in the Fe deficiency response, such as iron-regulated transporter 1 (IRT1; Vert et al., 2002), ferric-chelate oxidase reductase (FRO2; Connolly et al., 2003) CYP82C4 (Murgia et al., 2011), ferroportin/ironregulated (IREG2; Morrissey et al., 2009) metal tolerance protein (MTP3; Arrivault et al., 2006)(Table 2); viceversa, they show no significant correlation with the ferritin genes since their correlation values fall within the $[-0.3+0.02]$ range (data not shown).

According to such results, the AtP4H genes could be divided into three classes:

Class 1: positive or negative correlation with metabolic genes only (At3g28490, At2g43080, and At5g18900).

Class 2: positive correlation with Fe-related genes and positive or negative correlation with metabolic genes (At2g17720 and At3g06300, beside the positive control At3g13610).

Class 3: no significant correlation (positive or negative) with any of the genes tested (At1g20270, At4g33910, At5g66060, At4g35820).

Genes in class 1 might be not involved in the plant response to improve Fe uptake and trafficking in order to alleviate Fe deficiency symptoms.

Genes in class 2 might be the ones linking the stimulation of Fe deficiency response with the metabolic adaptations triggered by Fe deficiency (Figure 1) whereas genes in class 3 might contain the candidate Fe sensor(s) (Figure 1).

Regarding the genes in class 2 , it is interesting to notice that beside with the Fe-related genes, the positive control At3g13610
Table 1 | List of genes for which the correlation analysis with 2'-OG-dependent dioxygenases has been performed.

\begin{tabular}{|c|c|c|c|}
\hline \multicolumn{2}{|c|}{ Fe homeostasis genes } & \multicolumn{2}{|c|}{ Metabolic genes } \\
\hline IRT1 & At4g19690 & HXK1 & At4g29130 \\
\hline IRT2 & At4g19680 & HXK2 & At2g19860 \\
\hline $\mathrm{AHA} 2$ & At4g30190 & HXK4 & At3g20040 \\
\hline NAS1 & At5g04950 & HKL1 & At1g50460 \\
\hline NAS2 & At5g56080 & HXL3 & At4g37840 \\
\hline NAS3 & At1g09240 & PPC1 & At1g53310 \\
\hline NAS4 & At1g56430 & PPC2 & At2g42600 \\
\hline CYP82C4 & At4g31940 & PPC3 & At3g14940 \\
\hline IREG2 & At5g03570 & PPC4 & At1g68750 \\
\hline MTP3 & At3g58810 & PGK & At1g79550 \\
\hline Popeye & At3g47640 & PGK1 & At3g12780 \\
\hline Brutus & At3g18290 & $\mathrm{LDH}$ & At4g17260 \\
\hline NRAMP3 & At2g23150 & ENO1 & At1g74030 \\
\hline NRAMP4 & At5g67330 & ENOC & At2g29560 \\
\hline FRO3 & At1g23020 & ENO2 & At2g36530 \\
\hline FRO7 & At5g49740 & iPGAM & At1g09780 \\
\hline FRD3 & At3g08040 & PGM & At1g78050 \\
\hline ILR3 & At5g54680 & PDC2 & At5g54960 \\
\hline YSL1 & At4g24120 & PDC3 & At5g01330 \\
\hline ZIF1 & At5g13740 & G6PD4 & At1g09420 \\
\hline VIT1 & At2g01770 & MMDH2 & At3g15020 \\
\hline Fer1 & At5g01600 & mal dehydr family & At3g53910 \\
\hline Fer2 & At3g11050 & mal dehydr family & At4g17260 \\
\hline Fer3 & At3g56090 & mal dehydr family & At5g58330 \\
\hline \multirow[t]{3}{*}{ Fer4 } & At2g40300 & $\mathrm{ADH} 1$ & At1g77120 \\
\hline & & ADH transcrip factor & At2g44730 \\
\hline & & ADH transcrip factor & At3g24490 \\
\hline
\end{tabular}

Genes in the left column are known to be involved in the Fe deficiency response, in regulation of Fe homeostasis or Fe trafficking. Genes in the right column are involved in glycolysis or in the consumption of pyruvate by non-OXOPHOS reactions; genes coding for the four isoforms of Arabidopsis phosphoenolpyruvate carboxylase (PEPC; PPC1, 2, 3, 4) have been also included. IRT, iron-regulated transporter; AHA, Arabidopsis $\mathrm{H}^{+}$-ATPase; NAS, nicotianamine synthase; CYP82C4, cytochrome P450 82C4; IREG, ferroportin/iron-regulated; MTP3, metal tolerance protein; NRAMP, natural resistance-associated macrophage protein; FRO, ferric-chelate oxidase reductase; FRD, ferric reductase defective; ILR, IAAleucine resistant; YSL, yellow stripe-like; ZIF, zinc induced facilitator; VIT, vacuolar iron transporter; Fer, ferritin; HXK1, hexokinase; HXL, hexokinase-like; PPC, phosphoenolpyruvate carboxylase; PGK, phosphoglyceratekinase; $L D H$, lactate dehydrogenase; ENO, enolase; ENOC, cytosolic enolase; iPGAM, phosphoglycerate mutase; PGM, phosphoglycerate/bisphosphoglycerate mutase; PDC, pyruvate decarboxylase; G6PD4, glucose-6-phosphate dehydrogenase; $M M D H$, mitochondrial malate dehydrogenase; mal dehydr family, malate dehydrogenase family; $A D H$, alcohol dehydrogenase.

is positively correlated with PPC3 and ENO1, the At3g06300 gene coding for $\mathrm{P} 4 \mathrm{H} 2$ (Tiainen et al., 2005) is positively correlated with PPC1, PPC3, ENO1, and also negatively correlated with a malate dehydrogenase family member whereas the At2g17720 gene coding 
Table 2 | Correlation analysis of Arabidopsis thaliana 2'-OG dioxygenase genes with genes involved in Fe deficiency response or with genes possibly involved in metabolic reprogramming during Fe deficiency.

\begin{tabular}{|c|c|c|c|c|c|c|c|c|c|c|c|c|c|c|c|}
\hline & \multirow[t]{2}{*}{ AGI code } & \multicolumn{2}{|c|}{$\begin{array}{c}\text { 2'-OG-dioxyg } \\
\text { At3g12900 }\end{array}$} & \multicolumn{2}{|c|}{$\begin{array}{c}\text { 2'-OG-dioxyg } \\
\text { At3g13610 }\end{array}$} & \multicolumn{2}{|c|}{$\begin{array}{c}\text { P4H-1 } \\
\text { At2g43080 }\end{array}$} & \multicolumn{2}{|c|}{$\begin{array}{c}\text { P4H-2 } \\
\text { At3g06300 }\end{array}$} & \multicolumn{2}{|c|}{$\begin{array}{c}\text { P4H-4 } \\
\text { At5g18900 }\end{array}$} & \multicolumn{2}{|c|}{$\begin{array}{c}\text { P4H-5 } \\
\text { At2g17720 }\end{array}$} & \multicolumn{2}{|c|}{$\begin{array}{c}\text { P4H-6 } \\
\text { At3g28490 }\end{array}$} \\
\hline & & lin & $\log$ & lin & $\log$ & lin & $\log$ & lin & $\log$ & lin & $\log$ & lin & $\log$ & lin & $\log$ \\
\hline IRT1 & At4g19690 & 0.69 & 0.23 & 0.74 & 0.69 & 0.21 & 0.21 & 0.54 & 0.55 & 0.10 & 0.11 & 0.36 & 0.53 & -0.02 & 0.05 \\
\hline AHA2 & At4g30190 & 0.22 & 0.09 & 0.67 & 0.69 & 0.35 & 0.31 & 0.76 & 0.71 & 0.18 & 0.22 & 0.76 & 0.79 & -0.07 & -0.07 \\
\hline IREG2 & At5g03570 & 0.73 & 0.42 & 0.79 & 0.59 & 0.26 & 0.22 & 0.64 & 0.55 & 0.23 & 0.31 & 0.41 & 0.51 & -0.02 & -0.08 \\
\hline MTP3 & At3g58810 & 0.77 & 0.27 & 0.83 & 0.70 & 0.30 & 0.37 & 0.62 & 0.66 & 0.16 & 0.23 & 0.40 & 0.58 & -0.04 & -0.10 \\
\hline HXK4 & At3g20040 & 0.25 & 0.22 & 0.32 & 0.25 & 0.24 & 0.27 & 0.29 & 0.37 & 0.26 & 0.02 & 0.17 & 0.26 & 0.72 & 0.06 \\
\hline HXL3 & At4g37840 & 0.01 & 0.05 & -0.06 & -0.03 & 0.13 & 0.05 & -0.08 & -0.06 & 0.32 & 0.11 & -0.05 & -0.06 & 0.66 & 0.35 \\
\hline PPC1 & At1g53310 & 0.03 & -0.06 & 0.41 & 0.55 & 0.19 & 0.22 & 0.60 & 0.63 & 0.03 & 0.10 & 0.73 & 0.70 & -0.10 & -0.06 \\
\hline PGK1 & At3g12780 & -0.13 & -0.11 & -0.50 & -0.41 & -0.67 & -0.69 & -0.49 & -0.46 & -0.66 & -0.74 & -0.33 & -0.30 & -0.09 & -0.03 \\
\hline ENO1 & At1g74030 & 0.14 & 0.03 & 0.72 & 0.59 & 0.37 & 0.31 & 0.67 & 0.59 & 0.12 & 0.06 & 0.54 & 0.49 & -0.05 & -0.01 \\
\hline iPGAM & At1g09780 & 0.07 & 0.03 & 0.47 & 0.51 & 0.04 & 0.10 & 0.54 & 0.56 & -0.21 & -0.32 & 0.61 & 0.62 & -0.07 & -0.03 \\
\hline Mal. d. fam. & At5g58330 & -0.19 & -0.23 & -0.56 & -0.49 & -0.70 & -0.67 & -0.61 & -0.61 & -0.59 & -0.60 & -0.47 & -0.47 & -0.08 & 0.02 \\
\hline
\end{tabular}

For each gene pair, the Pearson's correlation coefficient, from logarithmic or linear analysis, is reported. Coefficients with values $\geq 0.60$ or $\leq-0.60$ are highlighted in gray.

for $\mathrm{P} 4 \mathrm{H} 5$ is positively correlated with PPC1 and iPGAM (Zhao and Assmann, 2011).

Interestingly, PPC1 and PPC3 are mainly expressed in root tissues and their expression is affected by abiotic stress when compared with PPC2, which is considered to cover an housekeeping role (Sanchez et al., 2006), whereas ENO1 encodes the plastid-localized isoform of phosphoenolpyruvate (PEP)-ENO (Prabhakar et al., 2009); PEP is further metabolized to Pyr by pyruvate kinase (PK). PEP and Pyr represent essential precursors for anaerobic reaction. PEP is fed into the schikimate pathway, which is localized within the plastid stroma (Herrmann and Weaver, 1999) and which is essential for a large variety of secondary products. Pyr can also act as precursor for several plastid-localized pathways, among which the mevalonate-independent way of isoprenoid biosynthesis (Lichtenthaler, 1999). Plastid-MEP (2-Cmethyl-D-erythritol 4-phosphate) pathways might be responsible for the synthesis of a signal molecule putatively involved in the regulation of Fe homeostasis (Vigani et al., 2013).

Such analysis is preliminary and needs to be extended to all 2'-OG-dioxygenase gene family members. Genes candidate as Fe sensors can be further analyzed experimentally, insofar that loss-of function mutants lacking the "Fe sensing" function should display a Fe deficiency response, even in Fe-sufficient conditions (see Figure 1, upper panel, right).

\section{FUTURE DIRECTIONS}

Elucidation of nutrient sensing and signaling is a major issue in plant physiology and crop production, with potential impact in the design of new biofortification strategies for improving yields as well as the nutritional value of crops of interest (Murgia et al., 2012; Schachtman, 2012). In Arabidopsis, a major sensor of nitrate is the nitrate transporter NRT1.1, which is the first representative of plant "transceptors," thus indicating their dual nutrient transport/signaling function (Gojon et al., 2011). Transceptors, whose feature is that transport and sensing activity can be uncoupled, have been described in animals and yeasts (Thevelein and Voordeckers, 2009; Kriel et al., 2011) and more active transceptors have been postulated also in plants (Gojon et al., 2011).Three major global challenges faced by agriculture are food and energy production as well as environmental compatibility (Ehrhardt and Frommer, 2012). Advancements in the area of nutrient sensing and signaling can positively contribute solutions to all these three challenges and the extensive analysis of the complete $2^{\prime}$ OG-dioxygenase gene family, based on pilot analysis described in the present perspective, could be a novel way to pursue these advancements.

\section{ACKNOWLEDGMENTS}

Gianpiero Vigani and Irene Murgia were supported by FIRB, Futuro in Ricerca 2012 (project code RBFR127WJ9) funded by MIUR. Gianpiero Vigani was further supported by "Dote Ricerca": FSE, Regione Lombardia. We are grateful to Prof. Shimizu BunIchi (Graduate School of Life Science, Toyo University, Japan) for kindly providing Arabidopsis At3g13610 KO mutant seeds and to Luca Mizzi (Milano University, Italy) for developing the programs to compute the correlation coefficients. 


\section{REFERENCES}

Arrivault, S., Senger, T., and Krämer, U. (2006). The Arabidopsis metal tolerance protein AtMTP3 maintains metal homeostasis by mediating $\mathrm{Zn}$ exclusion from the shoot under $\mathrm{Fe}$ deficiency and Zn oversupply. Plant J. 46, 861-879. doi: 10.1111/j.1365313X.2006.02746.x

Beekwilder, J., van Leeuwen, W., van Dam, N. M., Bertossi, M., Grandi, V. Mizzi, L., et al. (2008). The impact of the absence of aliphatic glucosinolates on insect herbivory in Arabidopsis. PLoS ONE 3:e2068. doi: 10.1371/journal.pone.0002068

Berri, S., Abbruscato, P., FaivreRampant, O., Brasiliero, A. C. M., Fumasoni, I., Mizzi, L., et al. (2009). Characterization of WRKY co-regulatory networks in rice and Arabidopsis. BMC Plant Biol. 9:120. doi: 10.1186/1471-2229-9-120

Brahimi-Horn, M. C., Chiche, J., and Pouyessegur, J. (2007). Hypoxia signalling controls metabolic demand. Curr. Opin. Cell Biol. 19, 223-229. doi: 10.1016/j.ceb.2007.02.003

Connolly, E., Campbell, N. H., Grotz, N., Prichard, C. L., and Guerinot, M. L. (2003). Overexpression of the FRO2 ferric chelate reductase confers tolerance to growth on low iron and uncovers posttranscriptional control. Plant Physiol. 133, 1102-1110. doi: 10.1104/pp.103.025122

Conte, S. S., and Walker, E. L. (2011). Transporter contributing to iron trafficking in plants. Mol. Plant 4, 464476. doi: 10.1093/mp/ssr015

Donnini, S., Prinsi, B., Negri, A. S., Vigani, G., Espen, L., and Zocchi, G. (2010). Proteomic characterization of iron deficiency responses in Cucumis sativus L. roots. BMC Plant Biol. 10:268. doi: 10.1186/1471-222910-268

Ehrhardt, D. W., and Frommer, W. B. (2012). New technologies for 21 century plant science. Plant Cell 24, 374 394. doi: 10.1105/tpc.111.093302

Giehl, R. F. H., Lima, J. E., and von Wiren, N. (2012). Localized iron supply triggers lateral root elongation in Arabidopsis by altering the AUX1-mediated auxin distribution. Plant Cell 24, 33-49. doi: 10.1105/tpc.111.092973

Gojon, A., Krouk, G., Perrine-Walker, F., and Laugier, E. (2011). Nitrate transceptor(s) in plants. J. Exp. Bot. 62, 2299-2308. doi: 10.1093/jxb/erq419

Gorzelanny, C., Pöppelmann, P., Pappelbaum, K., Moerschbacher, B. M., and Schneider, S. W. (2010). Human macrophage activation triggered by chitotriosidase-mediated chitin and chitosan degradation.
Biomaterials 31, 8556-8563. doi: 10.1016/j.biomaterials.2010.07.100

Herrmann, K. M., and Weaver, L. M. (1999). The shikimate pathway. Annu. Rev. Plant Physiol. Plant Mol. Biol. 50, 473-503. doi: 10.1146/annurev.arplant.50.1.473

Hieta, R., and Myllyharju, J. (2002). Cloning and characterization of a low molecular weight prolyl 4-hydroxylase from Arabidopsis thaliana. J. Biol. Chem. 277, 23965-23971. doi: 10.1074/jbc.M201865200

Hirsilä, M., Koivunen, P., Günzler, V., Kivirikko, K. I., and Myllyharju, J. (2003). Characterization of the human prolyl 4-hydroxylases that modify the hypoxia-inducible factor. J. Biol. Chem. 278, 30772-30780. doi: 10.1074/jbc.M304982200

Ishimaru, Y., Kakei, Y., Shimo, H., Bashir, K., Sato, Y., Uozumi, N., etal. (2011). A rice phenolic efflux transporter is essential for solubilizing precipitated apoplastic iron in the plant stele. J. Biol. Chem. 286, 24649-24655. doi: 10.1074/jbc.M111.221168

Jin, C. W., He, Y. F., Tang, C. X., Wu, P., and Zheng, S. J. (2006). Mechanisms of microbially enhanced $\mathrm{Fe}$ acquisition in red clover (Trifolium pratense L.). Plant Cell Environ. 29, 888-897. doi: 10.1111/j.13653040.2005.01468.x

Jin, C. W., Li, G. X., Yu, X. H., and Zheng, S. J. (2010). Plant $\mathrm{Fe}$ status affects the composition of siderophore-secreting microbes in the rhizosphere. Ann. Bot. 105, 835841. doi: 10.1093/aob/mcq071

Jin, C. W., You, G. Y., He, Y. F., Tang, C., Wu, P., and Zheng, S. J. (2007a). Iron deficiency-induced secretion of phenolics facilitates the reutilization of root apoplastic iron in red clover. Plant Physiol. 144, 278-285. doi: 10.1104/pp.107.095794

Jin, C. W., He, X. X., and Zheng, S. J. (2007b). The iron-deficiency induced phenolics accumulation may involve in regulation of $\mathrm{Fe}$ (III) chelate reductase in red clover. Plant Signal. Behav. 2, 327-332. doi: 10.4161/psb.2.5.4502

Jin, C. W., You, G. Y., and Zheng, S. J. (2008). The iron deficiencyinduced phenolics secretion plays multiple important roles in plant iron acquisition underground. Plant Signal. Behav. 3, 60-61. doi: 10.4161/psb.3.1.4902

Kai, K., Mizutani, M., Kawamura, N., Yamamoto, R., Tamai, M., Yamaguchi, H., etal. (2008). Scopoletin is biosynthesised via orthohydroxylation of feruloyl CoA by a 2-oxoglutarate-dependent dioxygenase in Arabidopsis thaliana. Plant J. 55, 989-999. doi: 10.1111/j.1365 313X.2008.03568.x

Kai, K., Shimizu, B., Mizutani, M. Watanabe, K., and Sakata, K. (2006). Accumulation of coumarins in Arabidopsis thaliana. Phytochemistry 67, 379-386. doi: 10.1016/j.phytochem.2005.11.006

Ken, Q., and Costa, M. (2006). Hypoxia-inducible factor-1 (HIF-1) Mol. Pharmacol. 70, 1469-1480. doi: 10.1124/mol.106.027029

Kobayashi, T., Nakanishi, H., Takahashi, M., Kawasaki, S., Nishizawa, N. K., and Mori, S. (2001). In vivo evidence that Ids3 from Hordeum vulgare encodes a dioxygenase that converts $2^{\prime}$ deoxymugineic acid to mugineic acid in transgenic rice. Planta 212, 864-871. doi: 10.1007/s004250000453

Kobayashi, T., and Nishizawa, N. K. (2012). Iron uptake, translocation, and regulation in higher plants. Annu. Rev. Plant Biol. 63, 131152. doi: 10.1146/annurev-arplant042811-105522

Kriel, J., Haesendonckx, S., RubioTexeira, M., Van Zeebroeck, G., and Thevelein, J. M. (2011). From transporter to transceptor: signalling from transporter provokes re-evaluation of complex trafficking and regulatory control. Bioessays 33, 870-879. doi: 10.1002/bies.201100100

Lan, P., Li, W., Wen, T. N., Shiau, J. Y., Wu, Y. C., Lin, W., et al. (2011). iTRAQ protein profile analysis of Arabidopsis roots reveals new aspects critical for iron homeostasis. Plant Physiol. 155, 821-834. doi 10.1104/pp.110.169508

Li, J., Wu, X., Hao, S., Wang, X., and Ling, H. (2008). Proteomic response to iron deficiency in tomato root. Proteomics 8, 2299-2311. doi: 10.1002/pmic.200700942

Lichtenthaler, H. K. (1999). The 1dideoxy-D-xylulose-5-phosphate pathway of isoprenoid biosynthesis in plants. Annu. Rev. Plant Physiol. Plant Mol. Biol. 50, 47-65. doi: 10.1146/annurev.arplant.50.1.47

López-Millán, A. F., Grusak, M. A., and Abadía, J. (2012). Carboxylate metabolism change induced by Fe deficiency in barley, a strategy II plant species. J. Plant Physiol. 169, 1121-1124. doi: 10.1016/j.jplph.2012.04.010

López-Millán, A. F., Morales, F. Andaluz, S., Gogorcena, Y., Abadia, A., De Las Rivas, J., et al. (2000). Responses of sugar beet roots to iron deficiency. Changes in carbon assimilation and oxygen use.
Plant Physiol. 124, 885-898. doi: 10.1104/pp.124.2.885

Menges, M., Dóczi, R., Okrész, L., Morandini, P., Mizzi, L., Soloviev, M., et al. (2008). Comprehensive gene expression atlas for the Arabidopsis MAP kinase signalling pathways. New Phytol. 179, 643-662. doi: 10.1111/j.1469-8137.2008.02552.x

Møldrup, M. E., Salomonsen, B., and Halkier, B. A. (2012). Engineering of glucosinolate biosynthesis: candidate gene identification and validation. Methods Enzymol. 515, 291-313. doi: 10.1016/B978-0-12394290-6.00020-3

Morrissey, J., Baxter, I. R., Lee, L., Lahner, B., Grotz, N., Kaplan, J., etal. (2009). The ferroportin metal efflux proteins function in iron and cobalt homeostasis in Arabidopsis. Plant Cell 21, 3326-3338. doi: 10.1105/tpc.109.069401

Murgia, I., Arosio, P., Tarantino, D., and Soave, C. (2012). Crops biofortification for combating "hidden hunger" for iron. Trends Plant Sci. 17, 47-55. doi: 10.1016/j.tplants.2011.10.003

Murgia, I., Delledonne, M., and Soave, C. (2002). Nitric oxide mediates ironinduced ferritin accumulation in Arabidopsis. Plant J. 30, 521-528. doi: 10.1046/j.1365-313X.2002.01312.x

Murgia, I., Tarantino, D., Soave, C., and Morandini, P. (2011). The Arabidopsis CYP82C4 expression is dependent on $\mathrm{Fe}$ availability and the circadian rhythm and it correlates with genes involved in the early Fe-deficiency response. J. Plant Physiol. 168, 894902. doi: 10.1016/j.jplph.2010.11.020 Myllyharju, J. (2003). Prolylhydroxylases, the key enzymes of collagen biosynthesis. Matrix Biol. 22, 15-24. doi: 10.1016/S0945053X(03)00006-4

Prabhakar, V., Löttgert, T., Gigolashvili, T., Bell, K., Flügge, U. I., and Häusler, R. E. (2009). Molecular and functional characterization of the plastid-localized phosphoenolpyruvate enolase (ENO1) from Arabidopsis thaliana. FEBS Lett. 583, 983-991. doi: 10.1016/j.febslet. 2009.02.017

Qi, H. H., Ongushaha, P. P., Myllyharju, J., Cheng, D., Pakkamen, O., Shi, Y., et al. (2008). Prolyl 4hydroxylation regulates Argonaute2 stability. Nature 455, 421-424. doi: 10.1038/nature07186

Rellán-Álvarez, R., Andaluz, S., Rodríguez-Celma, J., Wohlgrmuth, G., Zocchi, G., Álvarez- Fernández, A., et al. (2010). Changes in the proteomic and metabolic profiles of Beta vulgaris root tips in response to iron deficiency and resupply. BMC Plant 
Biol. 10:120. doi: 10.1186/1471-222910-120

Sanchez, R., Flores, A., and Cejudo, F. J. (2006). Arabidopsis phosphoenolpyruvate carboxylase genes encode immunologically unrelated polypeptides and are differentially expressed in response to drought and salt stress. Planta 223, 901-909. doi: 10.1007/s00425-005-0144-5

Schachtman, D. P. (2012). Recent advances in nutrient sensing and signalling. Mol. Plant 5, 1170-1172. doi: $10.1093 / \mathrm{mp} / \mathrm{sss} 109$

Schmidt, W., and Steinbach, S. (2000). Sensing iron-a whole plant approach. Ann. Bot. 86, 589-593. doi: 10.1006/anbo.2000.1223

Semenza, G. L. (2007). Hypoxiainducible factor 1 (HIF-1) pathway. Sci. STKE 2007, cm8. doi: $10.1126 /$ stke. $4072007 \mathrm{~cm} 8$

Shibuya, N., and Minami, E. (2001). Oligosaccharide signalling for defence responses in plant. Physiol. Mol. Plant Pathol. 59, 223-233. doi: 10.1006/pmpp.2001.0364

Thevelein, J. M., and Voordeckers, K. (2009). Functioning and evolutionary significance of nutrient transceptors. Mol. Biol. Evol. 26, 2047-2414. doi: 10.1093/molbev/msp 168

Thimm, O., Essigmann, B., Kloska, S., Altmann, T., and Buckhout, T. J. (2001). Response of Arabidopsis to iron deficiency stress as revealed by microarray analysis. Plant Physiol. 127, 1030-1043. doi: 10.1104/pp.010191

Tiainen, P., Myllyharju, J., and Koivunen, P. (2005).
Characterization of a second Arabidopsis thaliana prolyl 4hydroxylase with distinct substrate specificity. J. Biol. Chem. 280, 1142-1148. doi: 10.1074/jbc. M411109200

Vega, K., and Kalkum, M. (2012). Chitin, chitinase responses, and invasive fungal infections. Int. J. Microbiol. 2012:920459. doi: 10.1155/ 2012/920459

Vert, G., Grotz, N., Dédaldéchamp, F., Gaymard, F., Guerinot, M. L., Briat, J. F., etal. (2002). IRT1, an Arabidopsis transporter essential for iron uptake from the soil and for plant growth. Plant Cell 14, 1223-1233. doi: $10.1105 /$ tpc.001388

Vigani, G. (2012a). Discovering the role of mitochondria in the iron deficiency-induced metabolic responses of plants. J. Plant Physiol. $169,1-11$.

Vigani, G. (2012b). Does a similar metabolic reprogramming occur in Fe-deficient plant cells and animal tumor cells? Front. Plant Sci. 3:47. doi: 10.3389/fpls.2012.00047

Vigani, G., Donnini, S., and Zocchi, G. (2012). "Metabolic adjustment under $\mathrm{Fe}$ deficiency in roots of dicotiledonous plants," in Iron Deficiency and Its Complication, ed. Y. Dincer, (Hauppauge: Nova Science Publishers Inc.), 1-27. doi: 10.1016/j.jplph. 2011.09.008

Vigani, G., and Zocchi, G. (2009). The fate and the role of mitochondria in Fe-deficient roots of strategy I plants. Plant Signal. Behav. 5, 375-379. doi: $10.4161 /$ psb.4.5.8344
Vigani, G., Zocchi, G., Bashir, K., Philippar, K., and Briat, J. F. (2013). Signal from chloroplasts and mitochondria for iron homeostasis regulation. Trends Plant Sci. doi: 10.1016/j.tplants.2013.01.006 [Epub ahead of print].

Vlad, F., Spano, T., Vlad, D., Daher, F. B., Ouelhadi, A., and Kalaitzis, P. (2007a). Arabidopsis prolyl hydroxylases are differently expressed in response to hypoxia, anoxia and mechanical wounding. Physiol. Plant 130, 471-483. doi: 10.1111/j.13993054.2007.00915.

Vlad, F., Spano, T., Vlad, D., Daher, F. B., Ouelhadj, A., Fragkostefanakis, S., et al. (2007b). Involvement of Arabidopsis Prolyl 4 hydroxylases in hypoxia, anoxia and mechanical wounding. Plant Signal. Behav. 2, 368-369. doi: 10.4161/psb.2.5. 4462

Vogel, C., and Marcotte, E. M. (2012). Insights into the regulation of protein abundance from proteomic and transcriptomic analyses. Nat. Rev. Genet. 13, 227-232. doi: 10.1038/ nrg3185

Wan, J., Zhang, X. C., and Stacey, G. (2008). Chitin signaling and plant disease resistance. Plant Signal. Behav. 3, 831-833. doi: 10.4161/psb.3.10.5916

Zhao, Z., and Assmann, S. M. (2011). The glycolytic enzyme, phosphoglycerate mutase, has critical roles in stomatal movement, vegetative growth, and pollen production in Arabidopsis thaliana. J. Exp. Bot. 62, 5179-5189. doi: 10.1093/jxb/err223
Zocchi, G. (2006). "Metabolic changes in iron-stressed dicotyledonous plants," in Iron Nutrition in Plants and Rhizospheric Microorganisms, eds L. L. Barton and J. Abadía (Dordrecht: Springer), 359-370. doi: 10.1007/1-4020-4743-6_18

Conflict of Interest Statement: The authors declare that the research was conducted in the absence of any commercial or financial relationships that could be construed as a potential conflict of interest.

Received: 25 February 2013; paper pending published: 18 March 2013; accepted: 13 May 2013; published online: 31 May 2013.

Citation: Vigani G, Morandini $P$ and Murgia I (2013) Searching iron sensors in plants by exploring the link among 2 -OG-dependent dioxygenases, the iron deficiency response and metabolic adjustments occurring under iron deficiency. Front. Plant Sci. 4:169. doi: 10.3389/ fpls.2013.00169

This article was submitted to Frontiers in Plant Nutrition, a specialty of Frontiers in Plant Science.

Copyright (c) 2013 Vigani, Morandini and Murgia. This is an open-access article distributed under the terms of the Creative Commons Attribution License, which permits use, distribution and reproduction in other forums, provided the original authors and source are credited and subject to any copyright notices concerning any third-party graphics etc. 